\title{
Role of Lumbar Puncture in Late Onset Neonatal Sepsis
}

\section{Amit Kumar Das', Deepak Mishra ${ }^{2}$, Nitu Kumari Jha ${ }^{3}$, Rakesh Mishra ${ }^{4}$ and Soniya $\mathrm{Jha}^{5}$}

${ }^{1}$ Department of Paediatrics, Indira Gandhi Memorial Hospital, Male, Maldives

${ }^{2}$ Department of Paediatrics, Kanti Children's Hospital, Maharajgunj, Kathmandu, Nepal

${ }^{3}$ Department of Obstretics and Gynaecology, National Academy of Medical Sciences, Kathmandu, Nepal

${ }^{4}$ Department of Orthopaedics, NAMS, Kathmandu, Nepal

${ }^{5}$ Medical Officer, Ramlal Golchha Eye Hospital, Morang, Nepal

\section{Correspondence: \\ Amit Kumar Das \\ Department of Paediatrics, \\ Indira Gandhi Memorial Hospital, \\ Male, Maldives \\ E-mail: mitjlichsu@hotmail.com}

DOI: 10.3126/jnps.v39i3.28459

Submitted on: 2019-04-13

Accepted on: 2020-04-22

\section{Acknowledgements: None}

Funding: None

Conflict of Interest: None declared

Permission from IRB: Yes

To cite this article: Das AK, Mishra D, Jha NK, Mishra R, Jha S. Role of lumbar puncture in late onset neonatal sepsis. J Nepal Paediatr Soc. 2019;39(3):155-61.

\section{ABSTRACT}

Introduction: Neonatal sepsis is a clinical syndrome characterised by signs and symptoms of infection with or without accompanying bacteraemia in the first month of life. It is responsible for about $30-50 \%$ of the total neonatal deaths in developing countries. Neonatal sepsis can be divided into two sub-types depending upon whether the onset of symptoms is within the first 72 hours of life (Early Onset Neonatal Sepsis) or after 72 hours of life (Late Onset Neonatal Sepsis). Meningitis is an important complication of late onset neonatal sepsis.

Method: This was hospital based prospective observational study conducted among the neonates admitted with diagnosis of late onset neonatal sepsis in Neonatal Intermediate Care Unit (NIMCU) and Neonatal Intensive Care Unit (NICU) of a tertiary care referral centre in Kathmandu, Nepal from July 2016 to June 2017. The objective of this study was to evaluate the importance of performing lumbar puncture in neonates with LONS.

Results: $16.8 \%$ neonates with late onset neonatal sepsis were found to have meningitis. Among the neonates with meningitis $\mathrm{C}$ reactive protein was positive in $57.2 \%$ and negative in $42.8 \%$. Among the cases with abnormal CSF findings, blood culture was sterile in $85 \%$ cases and organism was isolated $15 \%$ cases. In $88.8 \%$ cases with positive blood culture, no meningitis was detected. Lumbar puncture was traumatic in one neonate $(0.8 \%)$ in first attempt. Apart from this no other complication of performing lumbar puncture was noted.

Conclusion: Lumbar puncture and CSF examination is mandatory in all cases with late-onset sepsis.

Keywords: CSF examination; late-onset sepsis; lumbar puncture

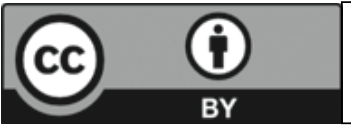

This work is licensed under creative common attribution 3.0 license 


\section{INTRODUCTION}

Neonatal sepsis is a clinical syndrome characterised by signs and symptoms of infection with or without accompanying bacteraemia in the first month of life. ${ }^{1}$ It encompasses various systemic infections of the newborn such as septicaemia, meningitis, pneumonia, arthritis, osteomyelitis and urinary tract infections. Superficial infections like conjunctivitis and oral thrush are not usually included under neonatal sepsis. Sepsis is one of the commonest causes of neonatal mortality. It is responsible for about $30-50 \%$ of the total neonatal deaths in developing countries. ${ }^{2,3}$ It is estimated that up to $20 \%$ of neonates develop sepsis and approximately $1 \%$ die of sepsis related causes. ${ }^{3}$

Neonatal sepsis can be divided into two sub-types depending upon whether the onset of symptoms is within the first 72 hours of life (Early Onset Sepsis, EONS) or after 72 hours of life (Late Onset Neonatal Sepsis, LONS). ${ }^{4}$ The source of infection in LONS is either nosocomial (hospital-acquired) or community-acquired and neonates usually present with septicaemia, pneumonia or meningitis. 5,6

Meningitis is an important complication of late onset neonatal infection. Various studies have shown cerebrospinal fluid (CSF) culture was positive in $1.3-22.5 \%$ of babies with LONS. ${ }^{7-13}$ These studies are in agreement that $15-50 \%$ of babies with meningitis (CSF culture positive) have negative blood cultures. The clinical features of septicaemia and meningitis often overlap; it is quite possible to have meningitis along with septicaemia without any specific symptomatology. This justifies the extra precaution of performing lumbar puncture (LP) in neonates with suspected sepsis. In EONS, lumbar puncture is indicated in the presence of a positive blood culture or if the clinical picture is consistent with septicaemia. It is not indicated if antibiotics have been started solely due to the presence of risk factors. In situations of late onset sepsis, LP should be done in all infants prior to starting antibiotics. However in a critically sick neonate lumbar puncture should be performed once the clinical condition stabilises. ${ }^{1}$ The primary argument in favour of universal inclusion of LP in septic workups (SWUs) is the observation that neonatal meningitis may occur in the presence of negative blood cultures.

\section{METHODS}

This was a hospital based prospective study conducted in a tertiary care referral centre for children, situated in Kathmandu, Nepal. The study was conducted after taking Institutional Review Committee clearance from the local ethical committee. We enrolled 125 neonates presenting after 72 hours of life and before 28 days, with signs and symptoms of clinical sepsis as per National Neonatology Forum criteria (NNF). The babies were admitted with diagnosis of LONS in Neonatal Intermediate Care Unit (NIMCU) and Neonatal Intensive Care Unit (NICU) of our institute from July 2016 to June 2017. As per NNF guidelines neonatal sepsis was categorised as follows:

(a) Blood culture positive cases (Confirmed / documented sepsis)

(b) Blood culture negative but sepsis screen positive cases (Probable sepsis)

(c) Blood culture negative and sepsis screen negative cases, but with clinical features compatible with sepsis (Clinical sepsis)- one of the following signs or symptoms: fever (temperature $>38^{\circ} \mathrm{C}$ ), hypothermia (temperature $<36^{\circ} \mathrm{C}$ ), decreased sucking, poor sucking or not sucking, lethargy, irritability, seizure, apnea, cough, respiratory distress or abdominal distention.

Sepsis screening as inclusion criteria is: A neonate was said to have sepsis if any two or more of the following investigations were abnormal: Total leukocyte count $<5000$ cells $/ \mathrm{mm}^{3}$, I/T ratio $>0.2$, ANC $<1800$ cells $/ \mathrm{mm}^{3}, \mathrm{~m}-\mathrm{ESR}>15 \mathrm{~mm}$ at the end of first hour, $\mathrm{C}$ reactive protein positive. Meningitis: greater than 30 leucocytes $/ \mathrm{mm}^{3}$ in CSF and / or organism isolated in CSF culture. Normal healthy neonates may have as many as 30 leucocytes $/ \mathrm{mm}^{3}$ (usually $<10$ ) in CSF. ${ }^{14}$

Investigations were sent which included complete blood count, random blood sugar, serum electrolytes (sodium and potassium) and blood culture and sensitivity. Urine analysis and chest Xray was done to detect the cause of fever. After informed written consent, LP was done and CSF was collected in two sterile vials: one for total count, differential count, protein and sugar and 
another for gram stain and culture. The vials were sent to the Emergency Laboratory for analysis. CSF was analysed as per standard laboratory methods in the Emergency Laboratory. The appearance of CSF was noted. For cell estimation, Neubauer counting chamber was used. For protein estimation, equal amount CSF was mixed with 3\% sulfosalicylic acid and turbidity was noted after 10 minutes which was compared with standard solution. For glucose estimation, Trinder's method was used. One $\mathrm{ml}$ of glucose reagent (glucose oxidase and peroxidase) was mixed with 10 microliter of CSF and incubated at $37^{\circ}$ Celsius for five minutes. The absorbance of the sample was read against the standard and glucose was calculated based on absorbance and concentration of caliber. For gram staining and culture, the CSF was sent to microbiology laboratory.

\section{RESULTS}

Out of 125 neonates, $70.4 \%$ of cases were of normal birth weight followed by $27.2 \%$ with low birth weight and $2.4 \%$ neonates were less than 1.5 $\mathrm{kg}$. There was male preponderance $(69 \%)$ with male : female ratio 2.2:1. Among the newborns enrolled in the study, 5.6\% were preterm, 92.0\% were full term and $2.4 \%$ were postdated. Twenty percent neonates were from Kathmandu valley and $80 \%$ were from outside valley. Ninety-three neonates $(74.4 \%)$ were delivered via SVD, two $(1.6 \%)$ were delivered via instrumental delivery and remaining 30 neonates $(24.0 \%)$ were delivered via LSCS.

As shown in figure above, poor feeding (89.6\%), fever / hypothermia (47.2\%), excessive / poor cry (40.8\%) and irritability / lethargy (33.6) were the common symptoms. Thirteen neonates (10.4\%) received some medical treatment while 112 $(89.6 \%)$ neonates didn't receive any medical treatment before reaching our hospital. As depicted in the figure above, CRP was positive in $31.2 \%$ $(39 / 125)$ of newborns. Abnormal haemoglobin level (42.4\%), abnormal total count (16.0\%), thrombocytopenia (12.8\%), low absolute neutrophil count $(4 \%)$ were the major laboratory abnormalities detected.

As seen in figure 3, blood culture was positive in 27 out of 125 cases, which accounts for $21.6 \%$ of total cases. In this study, 21 out of 125 neonates (16.8\%) with late onset neonatal sepsis were found to have meningitis. Organism was isolated from cerebrospinal fluid only in one case $(0.8 \%)$ and the isolated organism was Streptococcus pyogenes. The organism was sensitive to cotrimoxazole and erythromycin.

Staphylococcus aureus was the most predominant organism isolated in $66.7 \%(18 / 27)$ of newborns with a positive blood culture. Coagulase negative staphylococcus (CONS), next most common organism, was isolated in $18.5 \%$ of culture positive cases. The other organisms isolated were

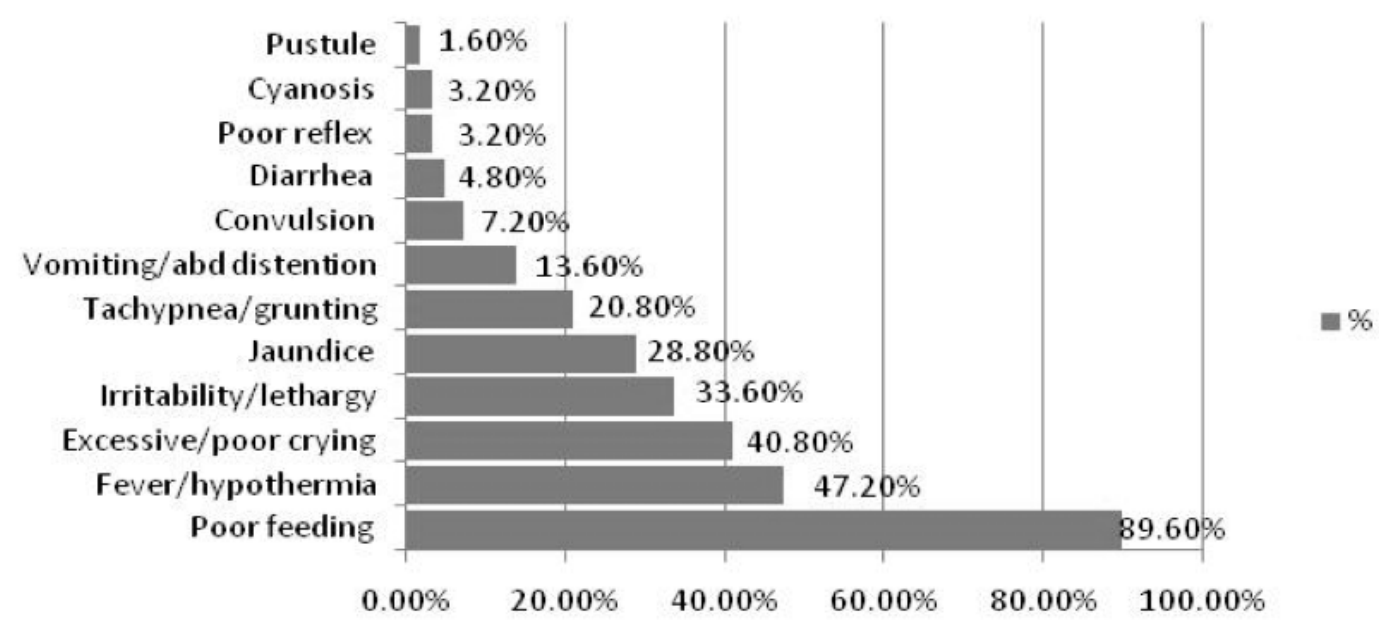

Figure 1. Prevalence of Hypertension 


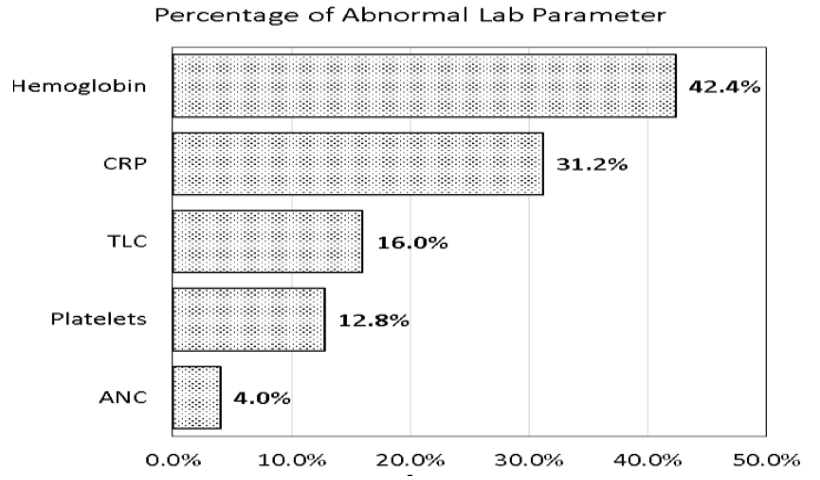

Figure 2. Distribution of study population by frequency of abnormality in respective laboratory parameter

Acenatobacter (11.1\%) and E. coli (3.7\%). Most of the isolated organisms showed sensitivity to cloxacillin (16/27), amikacin (15/27), ciprofloxacin (14/27), cefotaxime (11/27), cotrimoxazole (6/27), amoxyclox (6/27), cefixime (5/27) and amoxicillin (4/27). Lumbar puncture was traumatic in one neonate $(0.8 \%)$ in first attempt. Apart from this no other complication of performing lumbar puncture was noted.

\section{DISCUSSION}

In this study, a male predominance was observed. Some other studies have reported the incidence of male with neonatal sepsis to be as high as two folds as compared to females. Ibraheem $\mathrm{MF}$ et al. ${ }^{15}$ reported male to female ratio of 2.2:1. Similar study conducted among the neonates with sepsis by Shrestha NJ et al. ${ }^{16}$ had found male to female ratio of 1.7:1. All these studies support the result of our study and suggest the possibility of sex linked factor in host susceptibility and genetic susceptibility of male patients to infection. In our part of world, it might also be due to patriarchal system of our society which gives more emphasis to the male child.

In this study, 29.6\% (37/125) cases had birth weight less than $2500 \mathrm{gm}$ and $70.4 \%$ (88/125) babies had birth weight equal to or more than 2.5 $\mathrm{kg}$. In a study done in Paropakar Maternity hospital in 2011 there were $42.5 \%$ LBW babies and 57.5\% of appropriate weight babies. ${ }^{17}$ The lower incidence of LBW babies in our study might be due to inclusion of only $5.6 \%$ ( 7 cases) preterm babies. Furthermore the Nepal Multiple Indicator Cluster

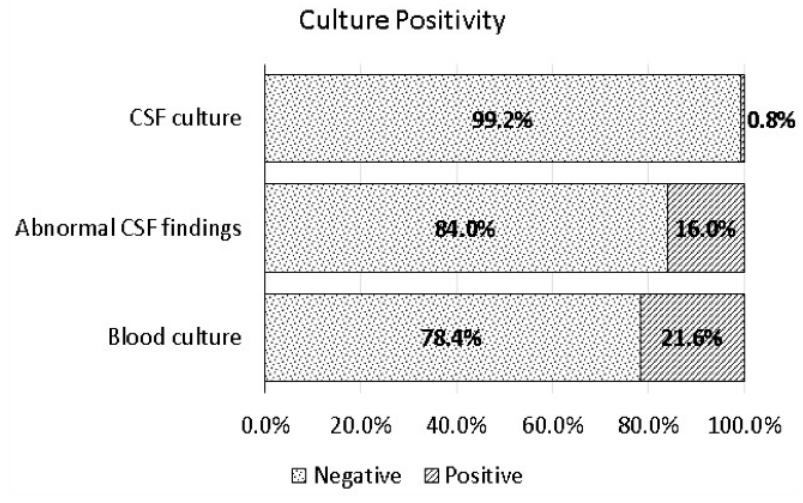

Figure 3. Distribution of study population by frequency of culture positivity in blood/CSF and abnormal CSF findings

Survey (MICS) 2014 shows that Percentage of most recent live births in the last two years weighing below 2,500 grams at birth is $24.2 \%$, which is also reflected in our study as having less percentage of low birth weight cases.

The symptoms with which the parents or caretakers presented in this study is almost comparable with the findings in study by Ibraheem FM et al..$^{15}$ and Ogunlesi TA et al. ${ }^{18}$ All these studies showed refusal to feed, fever, lethargy and respiratory distress to be the prominent features of presentation among the newborns with sepsis, which is comparable to our study. Similarly other symptoms like pustules and diarrhoea were below ten percent in all above mentioned studies which are in alignment with the findings of our study.

In this study, 13 neonates $(10.4 \%)$ had received some medical treatment while 112 (89.6\%) neonates didn't receive any medical treatment before reaching our institution. In a study done by Jumah DS et al. ${ }^{19} 26.66 \%$ had history of pretreatment before reaching the hospital while $73.33 \%$ had no any treatment before reaching the hospital. As $10.4 \%$ of cases in this study had already received antibiotics several days before they were referred, their blood and CSF culture were more likely to be sterile. In this study, the CRP was positive in $31.2 \%$ of cases $(39 / 125)$. In a study by Ahmed A. Khattab et al. ${ }^{13}$ CRP was positive in $90 \%$ cases. Among 20 cases with abnormal CSF findings CRP was positive in 11 cases $(55 \%)$ and negative in nine cases $(45 \%)$. 
In this study blood culture was positive in 27 out of 125 cases, which accounts for $21.6 \%$ of total cases. In a similar study conducted in Nepal by Shrestha $\mathrm{NJ}$ et al., ${ }^{16}$ organisms were isolated in $6.1 \%$ of the collected blood samples. In a study done by Chapagain et al., ${ }^{20} 14 \%$ of the suspected cases had positive blood culture. This study was conducted in a tertiary care hospital where most of the cases were referred from other hospitals and health facilities. Many of these babies $(10.4 \%)$ had already received antibiotics prior to referral rendering their blood culture sterile.

In this study $16 \%$ (20 out of 125 ) neonates with late onset neonatal sepsis were found to have abnormal CSF findings (greater than 30 leucocytes $/ \mathrm{mm}^{3}$ in CSF). In a study done by Ahmed A. Khattab et al. ${ }^{13}$, it was found that $23.3 \%$ cases with late onset neonatal sepsis had abnormal CSF findings with cells ranging between 80 and 26000 cells $/ \mathrm{mm}^{3}$. In this study organism was isolated from cerebrospinal fluid only in one case $(0.8 \%)$ and the isolated organism was Streptococcus pyogenes. The organism was sensitive to cotrimoxazole and erythromycin. In a study by Visser and Hall et al. ${ }^{7}$ $2.5 \%(5 / 193)$ CSF samples were positive. Schwersenski et al. ${ }^{8}$ conducted a study wherein 826 neonates underwent LPs, out of which 114 had LPs performed at greater than one week of age (late onset) and there were eight positive CSF cultures, but four were considered contaminants. In a similar study by Hristeva et al. ${ }^{9}$, 736 babies underwent LPs, of which 225 LPs were performed late (at $>48$ $\mathrm{h}$ of age), CSF cultures were positive in $1.3 \%$ $(4 / 310)$ of cases. In study by Ahmed AK et al. ${ }^{13}$ $20 \%$ (12 out of 60 neonates) presenting with lateonset neonatal septicaemia had positive CSF culture and most common organism in the CSF culture was Staphylococcus aureus.

In this study $16.8 \%$ (21 out of 125 ) neonates with late onset neonatal sepsis were found to have meningitis. Similar study done by Kaul et al ${ }^{12}$ reported that meningitis was found in $22.5 \%$ (23/102) neonates with LONS. In a study conducted by Visser and Hall et al. ${ }^{7}$ found that $2.5 \% \quad(5 / 193)$ CSF samples were positive. Schwersenski et al. ${ }^{8}$ and Hristeva et al. ${ }^{9}$ reported the prevalence of meningitis in late onset neonatal sepsis as $3.5 \%$ and $6.8 \%$ respectively, but the former excluded neonates more than 72 hour and less than seven days from their study and the latter delayed the LP procedure in all the babies who had respiratory distress. These variations in the prevalence could be explained also on the basis of the fact that meningitis was diagnosed in all these studies on the basis of CSF culture positivity alone.

In this study lumbar puncture was traumatic in one neonate $(0.8 \%)$ in first attempt. Apart from this no other complication of performing lumbar puncture was noted. This is in alignment with findings in other studies, wherein lumbar puncture has been found to be a safe procedure in neonates. In study by Visser VE et $\mathrm{al}^{7}$, Schwersenski et $\mathrm{al}^{8}{ }^{8}$ and Kumar et al. ${ }^{10}$ too no adverse effects of performing LP were found. Similarly, in the study by Stoll and colleagues $^{3}$ there was no difference in the risk of death between infants who underwent LP and who did not. In study by Hristeva et al. ${ }^{9}$ in which 736 babies underwent an LP, one case of probable posttraumatic meningitis was reported.

Blood culture was sterile in $81 \%$ cases $(17 / 21)$ and organism was isolated in $19 \%$ cases $(4 / 21)$ with meningitis. In $88.8 \%(24 / 27)$ cases with positive blood culture, no meningitis was detected. Also, in the only case in which organism was isolated in CSF culture, blood culture was sterile. In study conducted by Visser VE et al. ${ }^{7}, 24 \%(5 / 21)$ of septic babies with positive blood culture had meningitis and $15 \%$ baby with meningitis had concurrently negative blood cultures. They found 39 cases of meningitis based upon positive CSF cultures but in only 33 babies $(85 \%)$ was the organism also isolated from the blood. Thus in $15 \%$ of cases of meningitis the infecting organism would not have been identified by blood culture alone. In a study done by Kumar et al. ${ }^{10}$ it was found that all babies with meningitis had negative blood cultures. In a study carried out by Stoll et al. ${ }^{3} 7.2 \%$ of those with positive blood cultures had meningitis. 30\% (45/134) with meningitis had negative blood cultures. In a study done by Ahmed A. Khattab et al. ${ }^{13}$ it was found that among the 12 cases with positive CSF cultures, there were six cases $(50 \%)$ with negative blood cultures. In this study 20 cases with sterile cerebrospinal fluid had high CSF white blood cell counts and in one case in which organism was isolated from cerebrospinal was found that $2 / 60(3.3 \%)$ cases with CSF negative cultures had high CSF white blood cell counts. 
In this study only one out of 21 cases with meningitis $(4.76 \%)$ had abnormal cranial USG findings, in which bilateral third ventricles were dilated. In neonatal meningitis various sonographic findings range from echogenic and widened sulci, ventriculomegaly, ventriculitis, hydrocephalus, extra-axial fluid collections, cerebritis and brain abscess..$^{21}$ In a study done by Chowdary et al. ${ }^{22}$, a spectrum of sonographic abnormalities was observed which included echogenic sulci, focal or diffuse increase in parenchymal echoes, ventriculitis, ventriculomegaly with or without aqueductal block, subdural collection, parenchymal infarcts, abscess and subdural empyema. There were $5 \%(2 / 40)$ babies with meningitis with normal sonogram while encephalomalacia was seen in another 5\% (2/40) patients. An excellent correlation was observed between clinical profile, cerebrospinal fluid biochemistry and sonographic findings. ${ }^{22}$

\section{CONCLUSIONS}

There is a high incidence of neonatal meningitis among neonates with late-onset sepsis. Clinical features and laboratory abnormalities can't detect with sufficient sensitivity and specificity the babies having meningitis and neonatal meningitis frequently occurs in the absence of bacteraemia. If lumbar puncture is done only in cases in which CRP is positive or in which organism is isolated in blood culture or in which there is abnormal cranial USG findings, many cases with meningitis would be missed. Doing lumbar puncture in these babies is safe and their treatment with intravenous antibiotics is effective. Thus, lumbar puncture and CSF examination is mandatory in all cases with late-onset sepsis.

\section{REFERENCES}

1. Sankar MJ AR, Deorari AK, Paul VK. Sepsis in the newborn. Indian J. Pediatr. 2008;75(3):261-6. DOI:10.1007/ s12098-008-0056-Z

2. Bang AT BR, Baitule SB, Reddy MH, Deshmukh MD. Effect of home-based neonatal care and management of sepsis on neonatal mortality: field trial in rural India. The lancet. 1999;354(9194):1955-61. DOI:10.1016/ S0140-6736(99)03046-9

3. Stoll BJ HN, Fanaroff AA, Wright LL, Carlo WA, Ehrenkranz RA, Lemons JA, et. al. To tap or not to tap: high likelihood of meningitis without sepsis among very low birth weight infants. Paediatrics. 2004;113(5):1181-6. DOI: 10.1542/peds.113.5.1181

4. Paul VK, Bagga A. Ghai Essential Pediatrics. PaulVK. Neonatal sepsis. In: Vinod K Paul, Arvind Bagga, editors. GHAI Essential Pediatrics. 8th edition.CBS publisher and Distributer; 2013:163-165.

5. Baltimore RS. Neonatal nosocomial infections. Semin perinatol. 1998;22(1)25-32. WB Saunders. https://doi.org/ 10.1016/S0146-0005(98)80005-0

6. Wolach B. Neonatal sepsis: Pathogenesis and supportive therapy. Semin perinatol. 1997;21:28-38. DOI: https:// doi.org/10.1016/S0146-0005(97)80017-1

7. Visser VE, Hall RT. Lumbar punctures in the evaluation of suspected neonatal sepsis. J. Pediatr. 1980;96(6):1063-7. DOI:10.1016/s0022-3476(80)80643-3

8. Schwersenski J, McIntyre L, Bauer CR. Lumbar puncture frequency and cerebrospinal fluid analysis in the neonate. JAMA. 1991;145(1):54-8. DOI:10.1001/archpedi.1991.02160010058016

9. Hristeva L, Bowler I, Booy R, King A, Wilkinson AR. Value of cerebrospinal fluid examination in the diagnosis of meningitis in the newborn. Arch. Dis. 1993;69(5):514-7. DOI: 10.1136/adc.69.5_Spec_No.514

10. Kumar P, Sarkar S, Narang A. Role of routine lumbar puncture in neonatal sepsis. J Paediatr Child Health. 1995;31(1):8-10. DOI:10.1111/j.1440-1754.1995.tb02902.x 
11. Flidel-Rimon O, Leibovitz E, Eventov Friedman S, Juster-Reicher A, Shinwell ES. Is lumbar puncture (LP) required in every workup for suspected late-onset sepsis in neonates? Acta Paediatr. 2011;100(2):303-4. DOI: 10.1111/j.1651-2227.2010.02012.x

12. Kaul V, Harish R, Ganjoo S, Mahajan B, Raina SK, Koul D. Importance of obtaining lumbar puncture in neonates with late onset septicemia a hospital based observational study from north-west India. J Clin Neonatol. 2013;2(2): 83. DOI: $10.4103 / 2249-4847.116407$

13. Khattab AA, El-Lahony DM, Abdallah S. Should a neonate with possible late-onset sepsis always have lumbar puncture? Menoufia Med J. 2014;27(2):230. DOI: 10.4103/1110-2098.141649

14. Charles G. Prober, Roshni Mathew; Acute Bacterial Meningitis Beyond the Neonatal Period; Nelson textbook of Pediatrics. 20ed. Elsevier Health Sciences; 2015. pp 2940.

15. Ibraheem MF. Neonatal bacterial sepsis: risk factors, clinical features and short term outcome. Fac Med Baghdad. 2011;53(3):261-3. DOI: https://doi.org/10.32007/jfacmedbagdad.v3261-264\%25

16. Shrestha NJ, Subedi KU, Rai GK. Bacteriological profile of neonatal sepsis: a hospital based study. J Nepal Paediatr Soc. 2011; 31(1):1-5. DOI: https://doi.org/10.3126/jnps.v31i1.4158

17. Thapa B, Thapa A, Aryal DR, Thapa K, Pun A, Khanal S, et.al. Neonatal sepsis as a major cause of morbidity in a tertiary center of Kathmandu. J Nepal Med Assoc. 2013;52(192):549-56. . DOI: https://doi.org/10.31729/jnma.2424

18. Tinuade A. Predictors of Mortality in Neonatal Septicemia in an Under resourced Setting. J Natl Med Assoc. 2010;102(10):915-20. DOI: 10.1016/S0027-9684(15)30710-0

19. Duha S, Mohammed KH. Predictors of mortality outcome in neonatal Sepsis. MJBU. 2007;25(1):11-8. DOI: $10.33762 / \mathrm{mjbu} .2007 .48118$

20. Chapagain RH, Acharya R, Shrestha N, Giri BR, Bagale BB, Kayastha M. Bacteriological Profile of Neonatal Sepsis in Neonatal Intermediate Care Unit of Central Paediatric Referral Hospital in Nepal. J. Nepal Health Res. Counc. 2015;13(31):205-8. PMID: 27005713

21. Gupta N, Grover H, Bansal I, Hooda K, Sapire JM, Anand R, et al. Neonatal cranial sonography: ultrasound findings in neonatal meningitis-a pictorial review. QIMS, Quant Imaging Med Surg. 2017;7(1):123-31. DOI: 10.21037/qims.2017.02.01

22. Chowdhary V, Gulati P, Sachdev A, Mittal SK. Pyogenic meningitis: sonographic evaluation. Indian Pediatr. 1991;28(7):749-55. 